\section{Investigación pediátrica en Latinoamérica}

\section{Sr. Editor:}

El artículo publicado en la Revista por Carlos Castillo y col $^{1}$, aporta valiosos datos sobre la contribución de Latinoamérica a la investigación en pediatría. En su estudio usaron el campo de "afiliación" de la base de datos de PUBMED para decidir a que país correspondía cada estudio clínico en pediatría publicado entre 1996 y 2005.

Sin embargo, la metodología empleada podría subestimar la participación de nuestros países en la materia. El campo "afiliación" no tiene un formato estandarizado como los términos $\mathrm{MeSH}$, por lo que queda a criterio de cada autor la forma en la cual describe la misma. Valderas y col, demostraron que el nombre del país en el campo de afiliación sólo tiene 45,8\% de sensibilidad para identificar correctamente el país de origen. Usar el nombre del país en diversos idiomas, topónimos correspondientes a provincias y ciudades principales, y acrónimos de instituciones de salud y universidades, puede incrementar la sensibilidad a $88,1 \%{ }^{2}$.

Por otra parte, también se debe recordar que el campo "afiliación" sólo identifica a la del primer autor. Aunque es razonable pensar que éste tenga una injerencia trascendente en el estudio, esto no excluye que el mismo se ha desarrollado en varios países, o que su diseño y conducción corresponda a un país diferente del primer autor.

\section{Paula Otero $^{1}$, Eduardo Cuestas ${ }^{2}$, Fernando Ferrero ${ }^{1}$ Hospital Italiano de Buenos Aires, Argentina \\ ${ }^{2}$ Hospital Privado, Córdoba, Argentina ${ }^{3}$ Hospital de Niños Pedro de Elizalde, Buenos Aires, Argentina}

\section{Referencias}

1.- Castillo C, Pletikosic X, Pizarro F: Estudios Clínicos Controlados Aleatoreos, en la Pediatría de Latino América (1996-2005). Rev Chil Pediatr 2009; 80 (5): 420-6.
2.- Valderas JM, Mendivil J, Parada A, Losada-Yáñez M, Alonso J: Construcción de un filtro geográfico para la identificación en PubMed de estudios realizados en España. Rev Esp Cardiol 2006; 59 (12): 1244-51.

\section{Respuesta a observaciones de nuestro trabajo "Estudios clínicos controlados, aleatoreos en la Pediatría de Latino América (1996-2005)}

Nos parece muy interesante la información de los Drs. Otero, Cuesta y Ferrero acerca de nuestra publicación sobre el grado de sensibilidad de buscar la afiliación de los autores a través del campo respectivo en la base de datos de PUBMED.

Sin tener esa información que comentan, como información adicional a la afiliación, durante la búsqueda de los datos comunicados, efectuamos una exhaustiva búsqueda extra, revisando la base de datos LILACS y directamente en las Revistas de corriente principal de los países estudiados durante los años analizados y en las principales Revistas con índice de impacto de circulación internacional tanto pediátricas generales (ej: J Pediatr, Pediatrics, Pediatr Res, Eur J Clin Pediatr, Acta Paediatr, Early Hum Develop), como de áreas específicas del conocimiento o de la Pediatría (ej: JAMA, Pediatr Infect Dis, J Pediatr Gastroenterol Nutr, Br J Nutr, Pediatr Pulmonol) (página 421).

La mayor dificultad la encontramos con estudios efectuados y financiados en Latinoamérica por Universidades o profesionales de Estados Unidos o Europa, pero en los que no había referencia directa entre las afiliaciones a la participación de profesionales o instituciones del país donde se efectuó el estudio. Estos estudios no fueron considerados, al ser considerados una actividad académica de esos centros extranjeros.

Dr. Carlos Castillo D. El Editor 